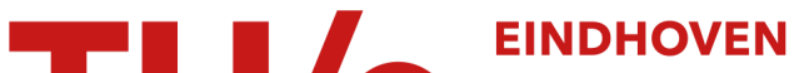 UNIVERSITY OF TECHNOLOGY
}

\section{Simplified ultrasonic damage detection in fluid filled pipes}

Citation for published version (APA):

Vogelaar, B. B. S. A., \& Golombok, M. (2017). Simplified ultrasonic damage detection in fluid filled pipes. Journal of Nondestructive Evaluation, 36(4), [63]. https://doi.org/10.1007/s10921-017-0443-3

DOI:

10.1007/s10921-017-0443-3

Document status and date:

Published: 01/12/2017

\section{Document Version:}

Publisher's PDF, also known as Version of Record (includes final page, issue and volume numbers)

\section{Please check the document version of this publication:}

- A submitted manuscript is the version of the article upon submission and before peer-review. There can be important differences between the submitted version and the official published version of record. People interested in the research are advised to contact the author for the final version of the publication, or visit the $\mathrm{DOI}$ to the publisher's website.

- The final author version and the galley proof are versions of the publication after peer review.

- The final published version features the final layout of the paper including the volume, issue and page numbers.

Link to publication

\section{General rights}

Copyright and moral rights for the publications made accessible in the public portal are retained by the authors and/or other copyright owners and it is a condition of accessing publications that users recognise and abide by the legal requirements associated with these rights.

- Users may download and print one copy of any publication from the public portal for the purpose of private study or research.

- You may not further distribute the material or use it for any profit-making activity or commercial gain

- You may freely distribute the URL identifying the publication in the public portal.

If the publication is distributed under the terms of Article $25 \mathrm{fa}$ of the Dutch Copyright Act, indicated by the "Taverne" license above, please follow below link for the End User Agreement:

www.tue.nl/taverne

\section{Take down policy}

If you believe that this document breaches copyright please contact us at:

openaccess@tue.nl

providing details and we will investigate your claim. 


\title{
Simplified Ultrasonic Damage Detection in Fluid-Filled Pipes
}

\author{
Bouko Vogelaar ${ }^{1,2}$ - Michael Golombok ${ }^{1,2}{ }^{(D}$
}

Received: 30 January 2017 / Accepted: 14 August 2017 / Published online: 21 August 2017

(C) The Author(s) 2017. This article is an open access publication

\begin{abstract}
The location and extent of damage in a pipe can be remotely determined from weld and internal damage reflections using a single acoustic emitter/sensor pair. The use of normalised reflections yields single numbers enabling long distance data collection techniques such as wireless hopping. The attenuation is twice as high for opposite inner and outer fluids (whether air and water, or water and air) as compared to identical inner and outer fluids. The absolute recorded signals in the water-filled pipe are attenuated by a factor two compared to the empty pipe. The axial length of detection is reduced by a half. The reduction of $>90 \%$ in sensors and the longer axial detection $(>10 \times$ current state-of- theart- technology) means that permanent fixed sensor pairs for whole pipelines are on the horizon of possibility. The greatest advantage is envisioned in submersed pipelines.
\end{abstract}

Keywords Structural health monitoring · Guided wave attenuation · Pipe damage $\cdot$ Welds $\cdot$ Water-filled

$\begin{array}{ll}\mathrm{L} & \text { Distance } \\ \mathrm{R} & \text { Reflection coefficient } \\ \mathrm{w} & \text { Wall thickness } \\ \alpha & \text { Attenuation coefficient } \\ \gamma & \text { Attenuation } \\ \delta & \text { Dimensionless damage depth parameter }\end{array}$

\section{Super/sub-scripts}

$\begin{array}{ll}0 & \text { Source } \\ \text { dam } & \text { Damage } \\ \text { dir } & \text { Direct } \\ \text { end } & \text { End } \\ \text { ref } & \text { Reference wave reflected from fixed element (either } \\ & \text { end or weld) } \\ \text { wel } & \text { Weld } \\ & \text { Alternative normalisation to weld reflection ampli- } \\ & \text { tude }\end{array}$

\section{Introduction}

Pipeline inspection for defects, including corrosion involves cumbersome and costly periodic visual inspections carried out by skilled personnel or remote operating vehicles. In areas where damage is suspected, visual inspections are followed by more advanced controls and methods, such as magnetic, ultrasonic, pressure testing, in-line pigs, and guided wave inspection [1]. In this last technique [2-6], acoustic waves are excited along the pipeline and the scattered signals are detected by distributed sensors mounted on the outer wall of the pipelines. Scattering occurs at non-axisymmetric features, which make guided waves suitable for damage 
detection via signal analysis $[7,8]$. In addition, attenuation occurs at strong impedance transitions from the pipe wall to the surrounding material, such as external coating, burial, or internal fluid [9-12].

Such testing is particularly good for unpiggable pipe monitoring - commercial examples include Teletest and G4 Wavemaker systems. As indicated above, guided wave monitoring is manpower intensive requiring successive placements of the coupling collars of sensors round the pipe. Each collar contains up to 16 signal sensors. We have shown that this can be reduced by $>90 \%$ and also made to work over a much longer pipe length [13-15] thus simplifying the overal configuration of such a detection system. This used the simplest acoustic mode, the so-called $\mathrm{T}(0,1)$ which has been shown to predominate in pipe transmission and to be dispersion free. An additional advantage (explained below) is that internal signal normalisation removes the requirement for baseline subtraction which is a major source of uncertainty in current methods. A single number is generated which provides a metric for the internal damage. This has enabled development of a method to locate and assess internal wall damage over ca. $150 \mathrm{~m}$ pipeline with a single sensor emitter/detector pair using reflections from pipe ends. Compared to the current state of the art, the foregoing improvements represent a considerable simplification and enhancement. We have recently shown [16] that welds themselves can provide a good reference reflection signal obviating the requirement for a baseline subtraction which is normally required in other guided wave techniques. In addition the technique is also resilient for all but the sharpest of pipe bends.

In this study, we address the most important obstacle which needs to be overcome to enable in-service use. (The current techniques require that pipes and tanks are drained before testing.) Because of the higher viscosity of liquids compared to gases, it is expected that signal attenuation would be caused by fluid inside or outside the pipe. The former case refers to normal surface downstream petrochemical operations whereas the latter typically refers to subsea production and transport-this also includes risers, subsea manifolds and containment systems such as tanks. We show that in these more realistic situations, we can still detect defects, localise them and assess severity even with a spatially sparse set of sensor pairs. Section 2 gives background on previous work. Section 3 describes the experiment and analysis. Section 4 reports our results.

\section{Background}

A pipe acts as a cylindrical waveguide allowing longitudinal, torsional, and flexural wave types [2,3]. Wave attenuation and mode conversion occur at axial symmetric impedance transitions, such as welds and pipe ends. Studies in fluid-filled and surrounded pipes are abundant [16]. Leak detection in buried fluid-filled pipes [18,19] and nondestructive testing of immersed plates are also reported [20]. However as opposed to these leak studies, partial damage detection-i.e. without pipe cracks and leaks-in fluid-filled immersed pipes using guided waves have only been reported in a limited number of works [21,22]. Aristégui et al. [17] investigate guided wave propagation in pipes with liquid loading on both the inside and outside of the pipe. The attenuation of a mode is measured by comparing the amplitudes of successive echoes. Studies on the ultrasonic energy interaction between the waveguide and acoustic environment and cylindrical guided wave leakage to liquid loading are similar problems [23].

Rizzo et al. [24] used a non-contact inspection/monitoring approach with a laser for the wave generation and a conventional immersion transducer system for the detection of damage in a submerged plate. Pistone and Rizzo [25] expanded the work by replacing the laser with an immersed ultrasonic transducer array. The numerical and experimental studies of Razi and Taheri [26] on a vibration-based damage detection technique provided satisfactory results with respect to identification of an advancing notch, regardless of the fluid-type the pipe carried [i.e., compressible (air) or incompressible (water)]. They concluded that the variable damping introduced by the presence of fluid inside the pipe would not weaken the method's diagnostic capability.

$\mathrm{Na}$ and Kundu [21] used flexural guided waves for the detection of material removal from the outer wall of a 0.6 $\mathrm{m}$ long water-filled pipe submerged in water. Djili et al. [22] used a single transducer for the emission and reception leakyguided waves to detect and locate small notches on the outer surface of a filled tube immersed in water. However, the propagation distance of compressional $\mathrm{L}(0,2)$ modes was small. Alleyne et al. [27] reported that the torsional $\mathrm{T}(0,1)$ mode is generally preferable to the longitudinal $\mathrm{L}(0,2)$ mode for longrange screening of liquid-filled pipes. The torsional wave mode was chosen in the study of Kwun et al. [9], because it is immune to the presence of liquid product in the pipe. Løvstad and Cawley [28] state that the $\mathrm{T}(0,1)$ mode is unaffected by liquid present on the inside or outside of the pipe.

\section{Experiment}

\subsection{Pipe}

Experiments were performed on a 15 and $19 \mathrm{~m}$ long carbon steel pipe of outer diameter $80 \mathrm{~mm}$ and wall thickness $\mathrm{w}=2 \mathrm{~mm}$. This was fabricated from $6.3 \mathrm{~m}$ sections connected by I form tungsten inert gas welding. The pipe is located on a raised sequence of trellis support stands and sits within a larger PVC duct of $20 \mathrm{~m}$ length (Fig. 1a). This allows for water to be both on the inside and the outside of 

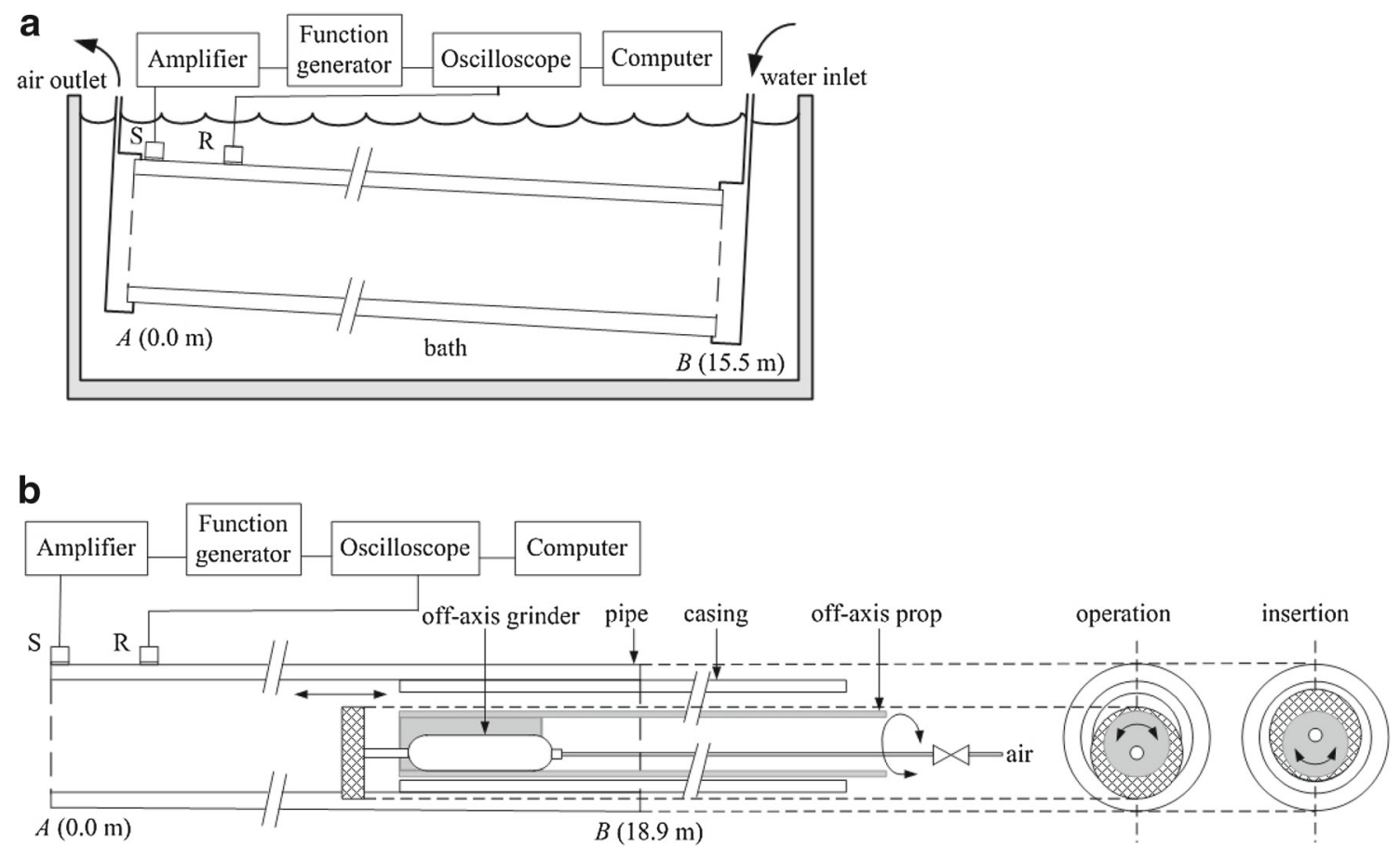

Fig. 1 Experimental layout of a pulse transmission set-up for extrinsic attenuation measurements. a Medium effect (air or water in- or outside the pipe). b Internal defect measurements

Table 14 Case studies for media inside and outside pipe

\begin{tabular}{lll}
\hline Case & Internal medium & External medium \\
\hline 1 & Air & Water \\
2 & Water & Water \\
3 & Water & Air \\
4 & Air & Air \\
\hline
\end{tabular}

the pipe. The tested pipe is slightly inclined, so that air bubbles can escape through the open outlet by buoyancy. The non-uniform hydraulic pressure is assumed to be negligible compared to shear.

Four situations are examined-see Table 1. In case 2, 3, and 4, the pipe is supported by two oblique M3-screws jutting out from the $\mathrm{V}$-shaped wedges. In case 1 the pipe tends to float, but is kept under water by putting weighted wedges on top of the pipe. Whether or not the pipe is filled or surrounded by water, the caps of the flow loop at the in- and outlet remain in place.

Internal wall damage is induced during a measurement program using a pneumatic grinder with its work spindle inserted plane-parallel to the axis of the pipe. The tool is modified with a $2.4 \mathrm{~m}$ long extended prop. For operation the extended prop is rotated in the casing off its axis to touch the inner wall of the pipe (Fig. 1b). The eccentric position of the axis of the grinder to the axis of the pipe ensures a round and flat part-circumferential material removal of the inner wall.
The radial movement mills a partial circumferential slot of the width of the grindstone in the inner wall and of depth $\mathrm{d}$. The axial movement then extends the axial dimension of the slot. This is repeated between each acoustic measurement. The depth increments in the pipe wall are $0.1 \mathrm{~mm}$ with a fixed axial extent of $10 \mathrm{~cm}$. We define the depth of damage by the fractional penetration of wall

$\delta=\frac{d}{w}$

The damage area is located $2 \mathrm{~m}$ from the pipe's remote end. Ten depth increments of $0.1 \mathrm{~mm}$ add up to a maximum damage depth of $1 \mathrm{~mm}$, which is $50 \%$ of the initial wall thickness (2 mm).

\subsection{Acoustics}

The acoustic set-up uses two piezoelectric shear wave transducers (Panametrics V1548) mounted on the pipe wall. The emitting and receiving transducers contain a piezoelectric shear element, sensitive to transverse displacement. The spectral amplitude at the applied frequency of $15 \mathrm{kHz}$ is $1 / 5$ th of the amplitude at the transducer center frequency of $100 \mathrm{kHz}$. The transducer element size is 1 inch diameter; their flat faces are clamped on the curved pipe wall without wedges or shoes at $0 \mathrm{~m}$ (source $\mathrm{S}$ ) and at $0.25 \mathrm{~m}$ (receiver R) from the pipe's near end (Fig. 1). The source-receiver offset 
a

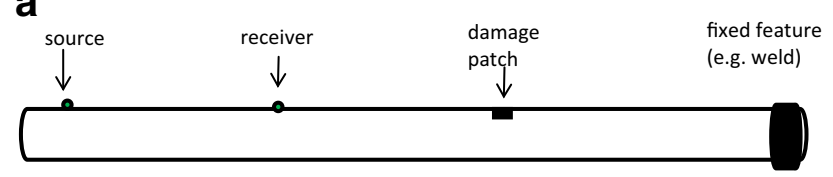

b

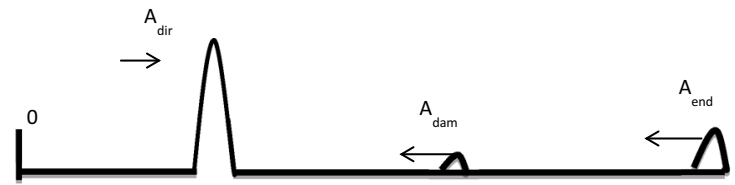

Fig. 2 Schematic of sequences received for analysis in time trace, a source and reflective elements on a pipe, $\mathbf{b}$ corresponding signal amplitudes

is thus about equal to the torsional wavelength. (The wavelength is the ratio of the shear wave velocity $(3.2 \mathrm{~km} / \mathrm{s}$ for carbon steel) and applied frequency.)

An arbitrary waveform generator feeds an amplifier with a single sine pulse of $15 \mathrm{kHz}$. The amplified signal is fed into the emitting shear transducer. This frequency is found to yield a strong signal. The associated torsional wavelength is near the pipe nominal circumference $(0.2 \mathrm{~m})$ and resonancelike performance at the torsional ring frequency occurs [13]. The detected signal is recorded over $50 \mathrm{~ms}$ by a digital oscilloscope with a sampling interval of $5 \mu \mathrm{s}$, thus containing $10^{4}$ data points per recording. The Nyquist frequency of 1 $\mathrm{MHz}$ prevents aliasing. Typically $4 \times 16$ successive recordings with a burst period of $1 \mathrm{~s}$ are captured and transferred to a computer for offline signal processing.

\subsection{Analysis}

\subsubsection{Reflection}

Figure $2 \mathrm{a}$ shows a schematic of the pipe with a emitter/detector sensor pair. Figure $2 b$ shows a schematic of the time trace measured at the receiver. There are three signals received in series:

1. The "direct" wave from the source with maximum $A_{\text {dir }}$.

2. A reflection from the damage spot with maximum $A_{d a m}$ (= 0 for undamaged pipe).

3. A reflection from a fixed feature. In the lab, this could be the end of the pipe with a reflection signal maximum $A_{\text {end }}$. For real pipes there is of course no such end available: in this study we make the comparison of both the end and a reflection from a weld with maximum $\mathrm{A}_{\text {wel }}$.

By ratioing the signals internally to the direct wave we obtain single parameters that do not require base subtraction from a separate trace. This also overcomes the problem of relatively strong torsional mode attenuation arising from surface damage, since both numerator and denominator are similarly affected in this case.

$R_{\text {ref }}=\frac{A_{\text {wel }}}{A_{\text {dir }}}$ or $\frac{A_{\text {end }}}{A_{\text {dir }}} ; \quad R_{\text {dam }}=\frac{A_{\text {dam }}}{A_{\text {dir }}}$

As damage increases, the second parameter $R_{\text {dam }}$ increases. Since the $\mathrm{T}(0,1)$ has a known single speed, the damage point can be located. The extent of the damage is given by the first parameter $\mathrm{R}_{\text {ref }}$ since the more damage that occurs the more damping occurs of the reference signal passing to and from the fixed characteristic (weld or end).

\subsubsection{Attenuation}

Even in an undamaged pipe, an acoustic signal attenuates as it progresses through a medium. There are two causes:

- intrinsic attenuation during transmission by absorption (conversion to heat and losses to the surrounding medium)

- extrinsic attenuation at reflections-mode conversion and scattering by welds, end, flanges etc.

We employ a point source for the generation of the fundamental torsional wave (circumferential motion) in a cylindrical geometry. The energy distribution by geometrical spreading causes attenuation in the near-field. We are primarily concerned here with the fundamental torsional wave which is non-dispersive. As a result the wave-packet spreading due to dispersion is zero in the far field. If the source emission is $\mathrm{A}_{0}$, then the measured amplitude $A$ over a distance $L$ from the source is described by a damped harmonic oscillator

$A=A_{0} e^{-\alpha L}$

with an attenuation coefficient $(\alpha)$ given by

$\alpha=-\frac{1}{L} \ln \left|\frac{A}{A_{0}}\right|$

The engineering definition of the attenuation $\gamma$ in decibels (dB) uses the base 10 logarithm of the ratio of signal power or intensity (proportional to $\mathrm{A}^{2}$ ) of the measured signal $\mathrm{A}$ and a reference signal $\mathrm{A}_{0}$ :

$\gamma=20 \log \left|\frac{A}{A_{0}}\right|$

As an example, a signal whose amplitude $\mathrm{A}$ is reduced to $10 \%$ of its initial value $A_{0}$ is said to be attenuated by $-20 \mathrm{~dB}$ (a decrease so a negative number). 

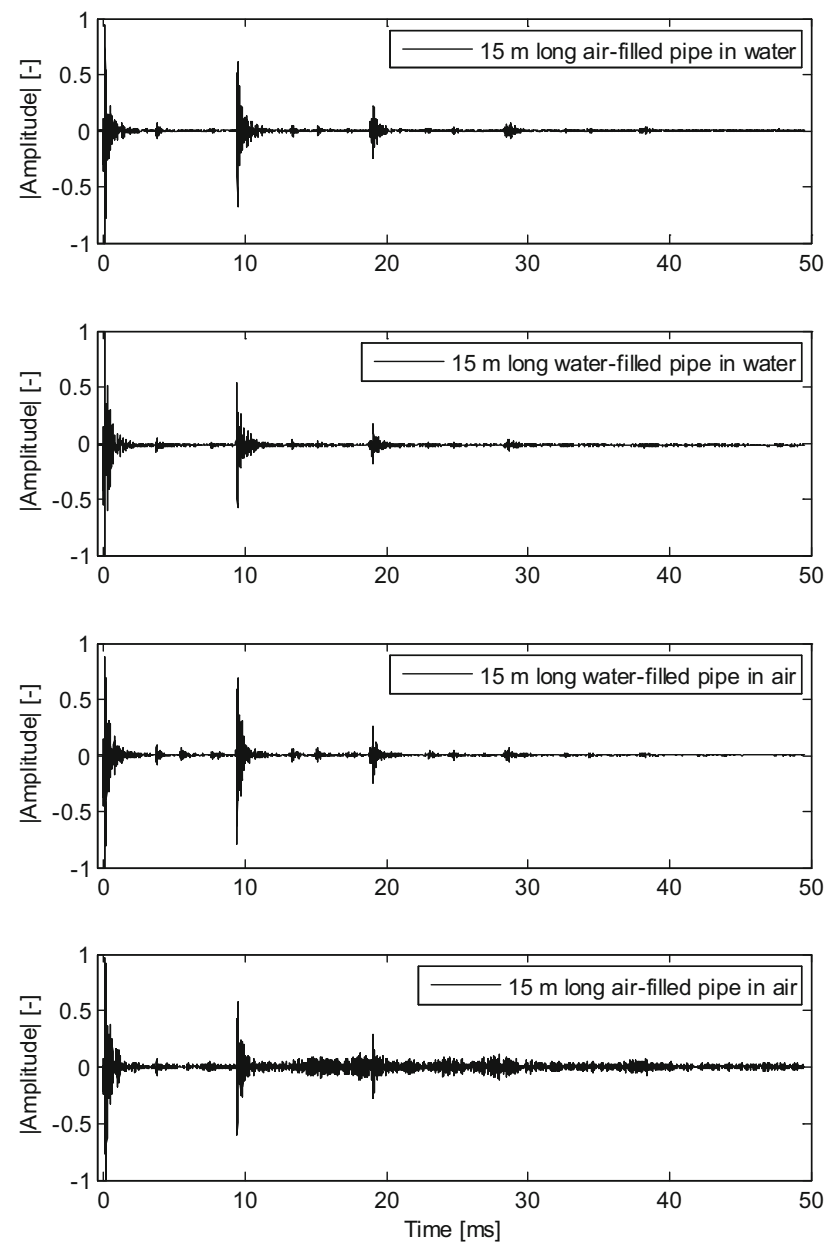

Fig. 3 Typical waveforms of a pulse-transmission experiment on a welded $15 \mathrm{~m}$ long carbon steel pipe with different internal and external media-ordered according to the cases in Table 1. The amplitudes are normalized to their maximum amplitude, which is from the direct wave at nearly zero time

\section{Results}

\subsection{Medium Effects}

Fluid effect on wave propagation is measured in a $15 \mathrm{~m}$ long pipe. Figure 3 shows typical traces for the possible permutations: the pipe filled with air or water; and the pipe surrounded by air or water. Five multiple reflections from the free end are detected by the single receiver. This corresponds to an axial detection single reflection in a pipeline over a distance of $150 \mathrm{~m}$ (five times two-way travel length).

The amplitudes are normalized to the maximum amplitude in each trace. As in Eq. 4, the measured amplitude is $A$ and the reference amplitude $\mathrm{A}_{0}=\mathrm{A}_{\mathrm{dir}}$, is the amplitude of the direct wave. Normalizing the amplitude to its absolute maximum amplitude per trace excludes loss of signal amplitude by weak coupling of the receiver to the pipe. The
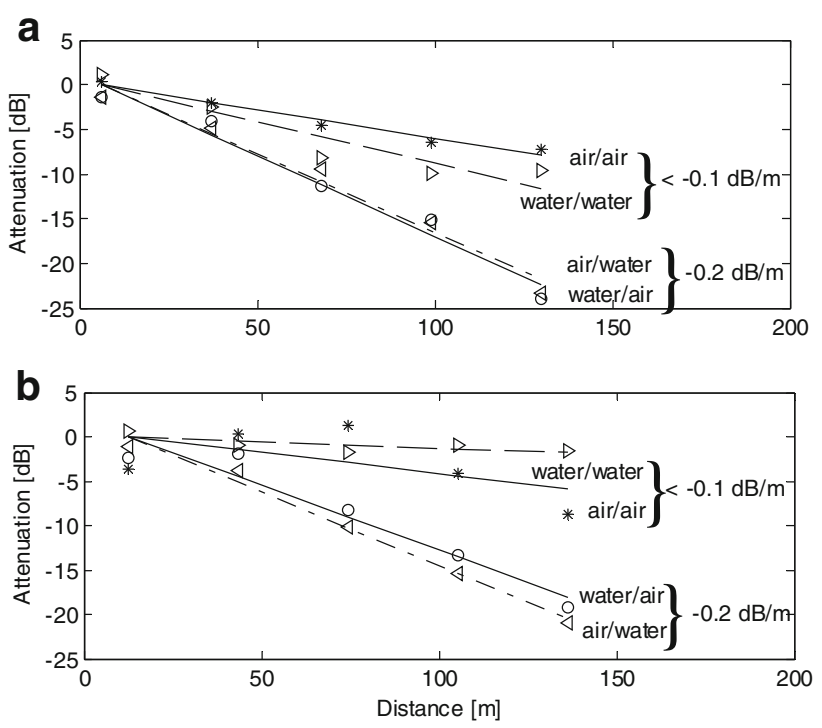

Fig. 4 Attenuation of the successive weld reflections for different internal/external media. The experimental data are normalized by the fit of the reference attenuation $(0 \mathrm{~dB})$ of the first weld at $6.3 \mathrm{~m}$ in $\mathbf{a}$ and of the second weld at $12.6 \mathrm{~m}$ in $\mathbf{b}$. The input is from Fig. 3

amplitude of the direct arrivals is then unity, and the absolute maximum amplitude of the reflected arrivals per trace can be used to calculate the attenuation coefficient from eq. 5. The direct wave arrives at nearly zero time. Several other multiple reflections are seen from the two welds at 6.3 and $12.6 \mathrm{~m}$. An extra mode is seen for the water-filled pipe in air between 5.5 and $6.0 \mathrm{~ms}$, which corresponds with the non-dispersive leak noise velocity, similar to the so-called Scholte wave [17].

Equal two-way travel time between the repeated signal envelopes dictates the location of reflection from the shear velocity of the torsional wave $(3.2 \mathrm{~km} / \mathrm{s}$ for carbon steel). Multiple reflections are separated by $2 \times 15 / 3.2 \mathrm{~ms}=10 \mathrm{~ms}$. Because the receiver is distant from the free end on the source side, the backward and forward propagating waves interfere and the reflection is smeared out in time. The first signal envelope after the direct arrival is the reflection from the first weld between 3.5 and $4.0 \mathrm{~ms}$. The $N$ th reflection of the first weld thus arrives $N \times 10 \mathrm{~ms}$ later, etc. A routine is written to automatically find the arrival time and peak amplitudes of the reflections from the first and the second weld.

The attenuation of the torsional mode is calculated by fitting the logarithm of the measured amplitudes of five successive reflections of the welds to a straight line. The effect of inner and outer fluids on the attenuation is shown in Fig. 4. The amplitude of the first arrival of the weld reflection is set as the reference amplitude. We observe that when the same fluid is inside and outside, then the attenuation coefficient is $-0.1 \mathrm{~dB} / \mathrm{m}$. For a different inner and outer fluid, the attenuation coefficient is twice as high. These values are comparable to the longitudinal attenuation of $-0.13 \mathrm{~dB} / \mathrm{m}$ 
in an air-filled pipe and $-0.22 \mathrm{~dB} / \mathrm{m}$ in a water-filled pipe from the experiments of Kwun et al. [29]. Typical attenuation coefficients in empty thick-walled bare pipes are -0.05 $\mathrm{dB} / \mathrm{m}$ for $30 \mathrm{kHz}$ torsional waves [9]. However it is not clear if this result would extend to very thick walls where the volume of pipe begins to match the volume of fluid it contains. For thicker pipes, the difference in attenuation is expected to taper off-this is the subject of current study.

The question then arises as to the physical origin of the effect described above. i.e. the reason that attentuation is twice as high for different inner and outer fluids compared to identical ones. These results suggest that it is easier to twist a pipe when the properties of the inner and outer fluid are identical than when they differ. This is related to the difference in resistance to shear at the inner and outer boundary of the thin wall. Different fluids at the inner and outer wall yield a stress gradient in the (membrane-like) wall, leading to the wave generation of flexural modes [13-15]. These fluidinduced vibrations add to the apparent high shear damping for different inner/outer fluids in flexible structures, such as our thin-walled pipe. The resulting differential stress when the fluids are different, leads to shear assymmetry at inner and outer wall resulting in $\mathrm{T}(0,1)$ losses by mode conversion. The tangential displacement is rather constant through the wall thickness for thin-walled pipes [30]. The dispersion and energy distribution of free waves in fluid-filled cylindrical shells is, however, strongly dependent upon the thickness of the shell wall, and on the ratio of the density of the shell material to the density of the containing fluid [31]. The shell stiffness is dependent on the standard dimension ratio ("SDR" = D/w). The tested pipe has a SDR of 40 and platelike behavior is expected. The imposed axisymmetric motion on the top and bottom boundary of a thin plate becomes nonsymmetric (stress releasing behavior) as the fluid density and viscosity at either boundary differ.

\subsection{Damage Detection}

The level of signal loss of the torsional wave mode caused by damage was assessed in laboratory experiments on a waterfilled pipe (with air outside). The attenuations are shown in Fig. 5 as a function of the damage depth for a water and air-filled pipe (cases 3 and 4 in Table 1) —again the external medium is air. The attenuation is calculated from Eq. 5 using measured end reflection amplitude $\mathrm{A}$ and reference direct amplitude $\mathrm{A}_{0}$. The damage depth is quantified by the percentage of the wall thickness (Eq. 1). The attenuation in both cases decreases roughly linearly with damage percent as shown by the trend lines. The slopes of the linear fits are identical, indicating that the attenuation coefficient per damage depth is independent of whether the pipe is dry or filled with water.

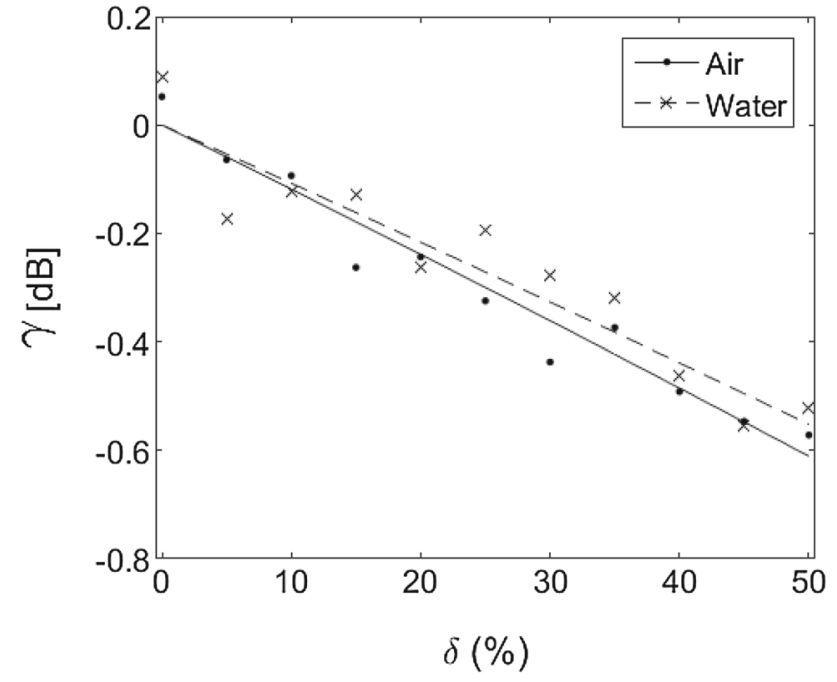

Fig. 5 Attenuation coefficient ( $\gamma$ Eq. 5) as a function of damage $\delta$ extent (Eq. 1) for air and water filled pipes (external environment air in both cases)

The absolute signal amplitude is however a factor two times lower for the water-filled pipe than for the empty pipe, so the signal-to-noise ratio of the latter is higher. A $6 \mathrm{~dB}$ drop in amplitude occurs as a result of water loading-see 4.1 above. Rose [32] also measured a $6 \mathrm{~dB}$ drop in the amplitude of non-axisymmetric Lamb waves as a result of water loading. Rose also obtained virtually identical results for a torsional mode in a dry and water loaded pipe using a shear horizontal electromagnetic acoustic transducer.

The increase in damage ( $\delta$ see Eq. 1$)$ thus reduces transmission of fixed reflected components (such as welds and free end reflections) as shown in Fig. 5 for empty and water-filled pipes respectively. This decrease in $\mathrm{R}_{\mathrm{ref}}$ is accompanied by the onset and increase of reflected damage amplitude $A_{d a m}$ because the damage itself partially reflects the direct wave [13]. As the damage increases, $R_{\text {dam }}$ (eq. 2) increases identically for empty and water-filled pipes (Fig. 6a). With only one (torsional) mode dominant in the reflected signal, localization of the damage is then straightforward from the difference in arrival times and the dispersion free torsional wave velocity.

Figure 3 showed that multiple reflections are obtained from welds. These weld signals can replace $A_{d i r}$ in the definition of an alternative parameter to $\mathrm{R}_{\mathrm{dam}}$ in Eq. 2. This is useful for distances further away from the sensors where (see Fig. 2) $A_{d i r}>>A_{d a m}$. In that case we can define another reflection parameter now normalised to the weld reflection (rather than the direct wave-see Eq. 2) by

$R_{d a m}^{\prime}=\frac{A_{d a m}}{A_{w e l}}$ 

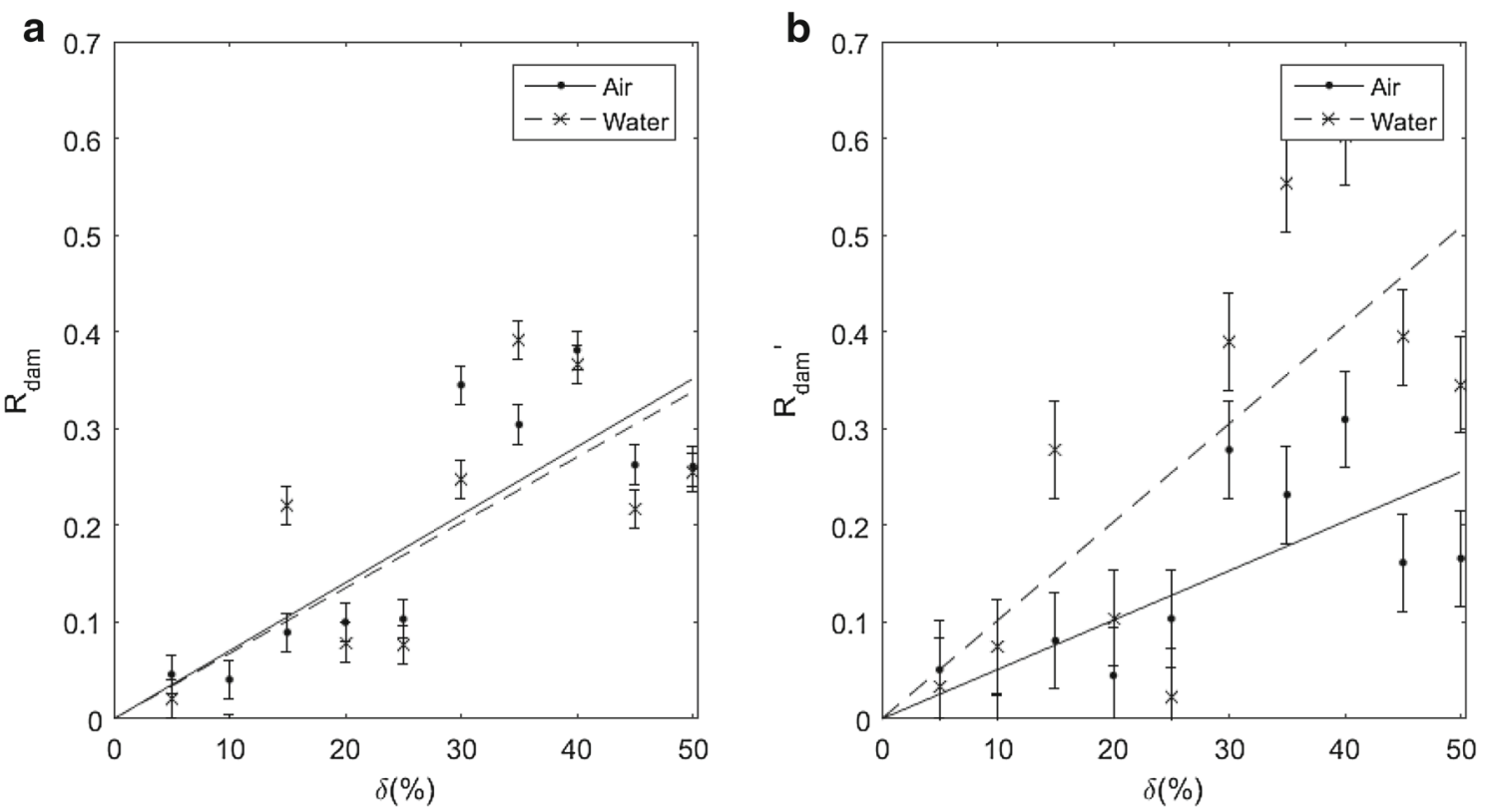

Fig. 6 Damage reflection coefficients as a function of damage extent $\delta$ (Eq. 1) for air and water filled pipes (external environment air in both cases) $\mathbf{a} \mathrm{R}_{\mathrm{am}}$ (Eq. 2) normalisation to "direct wave" and $\mathbf{b} \mathrm{R}_{\text {'dam }}$ (Eq. 6) normalisation to weld reference reflection

This is shown in Fig. $6 \mathrm{~b}$. Whereas $\mathrm{R}_{\mathrm{dam}}$ was insensitive to the medium, $R_{\mathrm{dam}}$ 'is more sensitive than $\mathrm{R}_{\mathrm{dam}}$ for a liquid filled pipe. The fits shown are trend lines: the relatively large amount of scatter derives from the fact that although severely dampened there are residual modes still present in our signal as we have previously shown [13-15]. $R_{\mathrm{dam}}$ ' displays much more scatter than $R_{\mathrm{dam}}$. This is because it is normalised to the weld reflected amplitude $A_{\text {wel }}$ which is much less than the direct wave $A_{\text {dir }}$ reflected from the end of the pipe. The error bars are therefore much greater for $\mathrm{R}_{\mathrm{dam}}$ '. In real applications, it is these weld reflections which are of course the relevant ones to consider- there is no detectable pipe end reflection. This result (using weld reflections) is a step toward passive monitoring of fluid transport pipes with commercial benefits since a sensor pair was demonstrated (in Fig. 4) to be able to cover at least 10 welds before the signal dies out. Since pipes are typically laid in lengths of $12 \mathrm{~m}$ this extends the range of the technique to the order of 100 $\mathrm{m}$. Thus rather than having a sequence of 2 rings of 16 sensors every ca. $10 \mathrm{~m}$, the new vision is a much more sparse system i.e. 1 sensor every ca. $100 \mathrm{~m}$. The value proposition of such a system becomes clear when one considers, as we have shown above, that the system is self-filtering and selfreferencing without the current requirement for time lapse base line subtraction. The effect of fluid has been shown to be minimal in systems where the internal and external media are the same. This opens up the possibility of accessing systems previously considered inaccessible such as subsea pipelines, seabed manifolds and storage systems-it now becomes economically feasible to continuously monitor such systems.

\section{Conclusions}

1. The fluid media inside and outside a pipe are the primary sources of attenuation determining the axial survival of a torsional wave used for detecting internal damage.

2. Identical inner and outer fluids (whether air or water) attenuate $-0.1 \mathrm{~dB} / \mathrm{m}$. This doubles when the inner and outer fluids are different. The torsional wave is thus attenuated twofold in a water-filled pipe compared to the empty, air-filled, situation (external environment air in both cases). The inspection range is reduced from ca. $150 \mathrm{~m}$ in straight empty pipes to ca. $75 \mathrm{~m}$ in water filled pipes under these circumstances.

3. The results demonstrate that the torsional wave is suitable for practical structural health monitoring of steel pipes to detect internal damage in liquid-filled welded pipes.

4. Other areas to which these results can be applied include flow induced vibrations in heat exchange channels, determination of internal damage in filled hydrocarbon tanks as well as scenarios to which guided waves could not be previously applied such as submerged risers, pipes and manifolds.

Funding This work was funded by Shell Global Solutions International B.V. under contract PT24225.

Open Access This article is distributed under the terms of the Creative Commons Attribution 4.0 International License (http://creativecomm ons.org/licenses/by/4.0/), which permits unrestricted use, distribution, and reproduction in any medium, provided you give appropriate credit to the original author(s) and the source, provide a link to the Creative Commons license, and indicate if changes were made. 


\section{References}

1. Liu, Z., Kleiner, Y.: State-of-the-art review of technologies for pipe structural health monitoring. IEEE Sens. J. 12, 1987-1992 (2012). doi:10.1109/JSEN.2011.2181161

2. Cawley, P., Alleyne, D.N.: The use of Lamb waves for the long range inspection of large structures. Ultrasonics 34, 287-290 (1996). doi:10.1016/0041-624X(96)00024-8

3. Lowe, M.J.S., Alleyne, D.N., Cawley, P.: Defect detection in pipes using guided waves. Ultrasonics 36, 147-154 (1998). doi:10.1016/ S0041-624X(97)00038-3

4. Rose, J.L.: A baseline and vision of ultrasonic guided wave inspection potential. J. Press. Vessel Technol. 124, 273-282 (2002). doi:10.1115/1.1491272

5. Su, Z., Ye, L., Lu, Y.: Guided Lamb waves for identification of damage in composite structures: a review. J. Sound Vib. 295, 753780 (2006). doi:10.1016/j.jsv.2006.01.020

6. Raghavan, A., Cesnik, C.E.S.: Review of guided-wave structural health monitoring. Shock Vib. Dig. 39, 91-114 (2007)

7. Grabowska, J., Palacz, M., Krawczuk, M.: Damage identification by wavelet analysis. Mech. Syst. Signal Process. 22, 1623-1635 (2008). doi:10.1016/j.ymssp.2008.01.003

8. Kim, C.-Y., Park, K.-J.: Mode separation and characterization of torsional guided wave signals reflected from defects using chirplet transform. NDT \& E Int. 74, 15-23 (2015). doi:10.1016/j.ndteint. 2015.04.006

9. Kwun, H., Kim, S.Y., Choi, M.S., Walker, S.M.: Torsional guidedwave attenuation in coal-tar-enamel-coated, buried piping. NDT \& E Int. 37, 663-665 (2004). doi:10.1016/j.ndteint.2004.05.003

10. Cobb, A.C., Kwun, H., Caseres, L., Janega, G.: Torsional guided wave attenuation in piping from coating, temperature, and largearea corrosion. NDT \& E Int. 47, 163-170 (2012). doi:10.1016/j. ndteint.2012.01.002

11. Kirby, R., Zlatev, Z., Mudge, P.: On the scattering of torsional elastic waves from axisymmetric defects in coated pipes. J. Sound Vib. 331, 3989-4004 (2012). doi:10.1016/j.jsv.2012.04.013

12. Leinov, E., Lowe, M.J.S., Cawley, P.: Investigation of guided wave propagation and attenuation in pipe buried in sand. J. Sound Vib. 347, 96-114 (2015). doi:10.1016/j.jsv.2015.02.036

13. Vogelaar, B., Golombok, M., Campman, X.: Pipe attrition acoustic locater (PAAL) from multi-mode dispersion analysis. Ultrasonics 68, 80-88 (2016). doi:10.1016/j.ultras.2016.02.009

14. Vogelaar, B., Golombok, M.: Quantification and localization of internal pipe damage. Mech. Syst. Signal Process. 78, 107-117 (2016). doi:10.1016/j.ymssp.2015.10.011

15. Vogelaar, B., Golombok, M.: Dispersion and attenuation by transmission, reflection, and mode conversion in welded pipes. Appl. Acoust. 110, 1-8 (2016). doi:10.1016/j.apacoust.2016.03.007

16. Vogelaar, B., Golombok, M.: Damage detection through pipe bends. J. Press. Vessel Technol. 139(051707), 1-7 (2017). doi:10. $1115 / 1.4037120$

17. Aristégui, C., Lowe, M.J.S., Cawley, P.: Guided waves in fluidfilled pipes surrounded by different fluids. Ultrasonics 39, 367-375 (2001). doi:10.1016/S0041-624X(01)00064-6
18. Muggleton, J.M., Brennan, M.J., Pinnington, R.J.: Wavenumber prediction of waves in buried pipes for water leak detection. J. Sound Vib. 249, 939-954 (2002). doi:10.1006/jsvi.2001.3881

19. Long, R., Lowe, M., Cawley, P.: Attenuation characteristics of the fundamental modes that propagate in buried iron water pipes. Ultrasonics 41, 509-519 (2003). doi:10.1016/S0041-624X(03)00166-5

20. Giurgiutiu, V., Cuc, A.: Embedded non-destructive evaluation for structural health monitoring, damage detection, and failure prevention. Shock Vib. Dig. 37, 83-105 (2005). doi:10.1177/ 0583102405052561

21. Na, W.-B., Kundu, T.: Underwater pipeline inspection using guided waves. J. Press. Vessel Technol. 124, 196-200 (2002). doi:10.1115/ 1.1466456

22. Djili, S., Benmeddour, F., Moulin, E., Assaad, J., Boubenider, F.: Notch detection in copper tubes immersed in water by leaky compressional guided waves. NDT \& E Int. 54, 183-188 (2013). doi:10. 1016/j.ndteint.2012.10.009

23. Rose, J.L., Cho, Y., Ditri, J.L.: Cylindrical guided wave leakage due to liquid loading. Rev. Prog. Quant. Nondestr. Eval. 13, 259-266 (1994)

24. Rizzo, P., Han, J.-G., Ni, X.-L.: Structural health monitoring of immersed structures by means of guided ultrasonic waves. J. Intell. Mater. Syst. Struct. 21, 1397-1407 (2010). doi:10.1177/ 1045389X10384170

25. Pistone, E., Li, K., Rizzo, P.: Noncontact monitoring of immersed plates by means of laser-induced ultrasounds. Struct. Health Monit. 5-6, 549-565 (2013). doi:10.1177/1475921713506767

26. Razi, P., Taheri, F.: A vibration-based strategy for health monitoring of offshore pipelines' girth-welds. Sensors 14, 17174-17191 (2014). doi:10.3390/s140917174

27. Alleyne, D.N., Vogt, T., Cawley, P.: The choice of torsional or longitudinal excitation in guided wave pipe inspection. Insight Non-Destr. Test. Cond. Monit. 51, 373-377 (2009). doi:10.1784/ insi.2009.51.7.373

28. Løvstad, A., Cawley, P.: The reflection of the fundamental torsional guided wave from pit clusters in pipes. NDT \& E Int. 46, 83-93 (2012). doi:10.1016/j.ndteint.2011.11.006

29. Kwun, H., Bartels, K.A., Dynes, C.: Dispersion of longitudinal waves propagating in liquid-filled cylindrical shells. J. Acoust. Soc. Am. 105, 2601-2611 (1999). doi:10.1121/1.426876

30. Pavlakovic, B., Lowe, M., Alleyne, D., Cawley, P.: Disperse: a general purpose program for creating dispersion curves. Rev. Prog. Quant. NDE 16, 185-192 (1997)

31. Fuller, C.R., Fahy, F.J.: Characteristics of wave propagation and energy distributions in cylindrical elastic shells filled with fluid. J. Sound Vib. 81, 501-518 (1982). doi:10.1016/ 0022-460X(82)90293-0

32. Rose, J.L.: Guided wave testing of water loaded structures. Mater. Eval. 61, 23-24 (2003) 